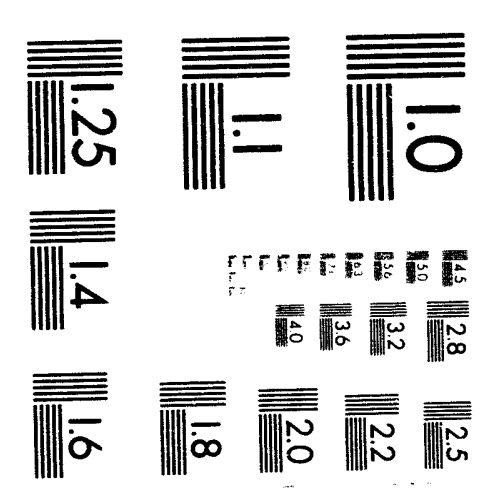



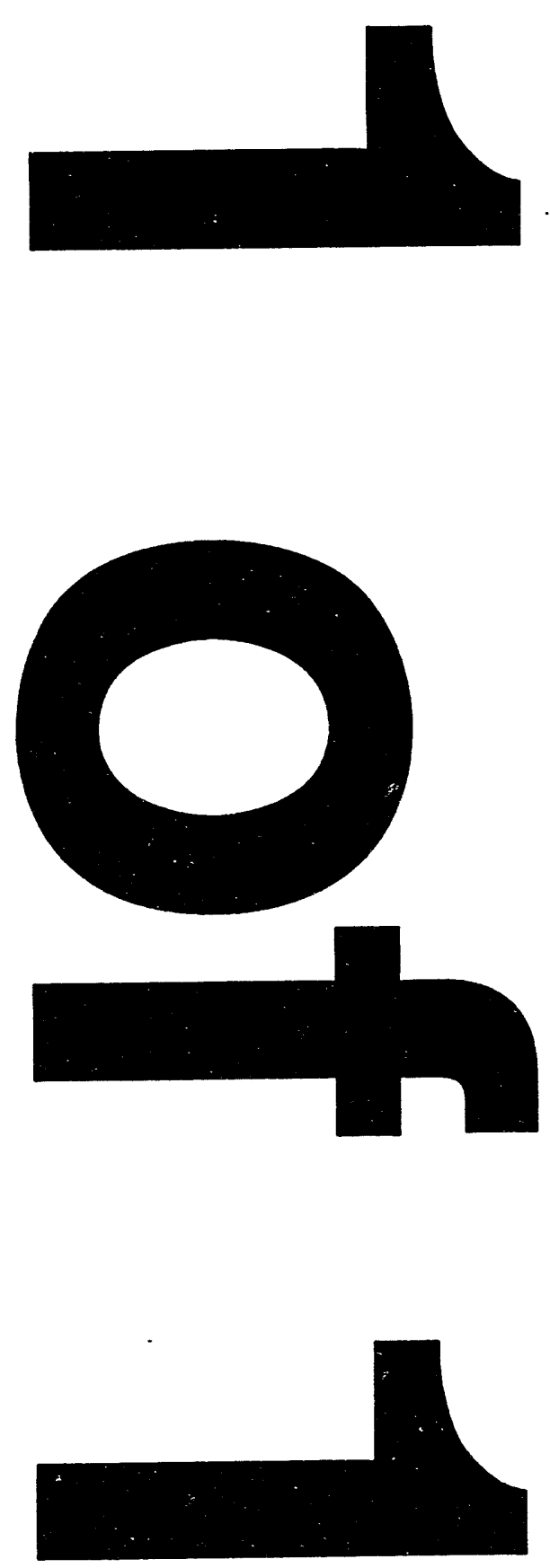
ANNUAL REPORT

DE-FG03-91ER20039

Department of Cell Biology, MRC-7

The Scripps Research Institute

10666 No. Torrey Pines Dr., La Jolla, CA 92037

The goals of this research are to identify the cis DNA sequence elements that are involved in regulating the expression of the $\alpha$ and $\beta$ subunits of $\beta$-conglycinin, a soybean seed storage protein. The basic approach has been to use transgenic plants to express chimeric genes comprising one of the two gene promoters ligated with the coding region of $\beta$-glucuronidase (GUS). Mutations were made within the promoters and the effects of the mutations on expression of the genes was determined. These studies were complemented by studies of the nuclear proteins that bound to the cis DNA sequences. An important goal of these studies has been to determine the molecular basis that controlled the different temporal and quantitative levels of expression of the two promoters.

Lessard et al (submitted manuscript) made block and linker scanning mutations in the $\alpha^{\prime}$ subunit gene promoter and demonstrated that what was conceived to be part of an enhancer element, in some mutant promoters, acted as a silencing or down-regulating sequence. Those sequences that were essential for high levels of gene expression included a region that bound Soybean Embryo Factor 4, and the RY element (for this promoter, CATGCAT). Sequences to which the SEF 3 factor bound were not essential for high levels of gene expression. Because the SEF 3 sequence was uniquely associated the $\alpha^{\prime}$ promoter, we had earlier suggested that this sequence was responsible for the high level, and temporally early expression of the $\alpha$ gene relative to the $\beta$-gene. The results of this study suggests that the role of the element may depend heavily upon the context of the sequence into which it is placed, including those that are far upstream. Concurrent with this study Lessard et al also developed $5^{\prime}$ terminal deletions of a $>1 \mathrm{~kb}$ promoter fragment from the $\beta$-gene and expressed the GUS gene in transgenic plants. This study demonstrated very clearly that there is a 'negative' element far upstream of the promoter, and that sequences between -553 and -72 contained quantitative elements of gene expression.

DrSTMBUTION OF THIS DOCUMFNT IS IBNLIMITEO

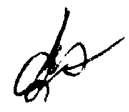


Page 2 of 5

We were quite disappointed to discover that none of the mutations developed during the course of this study affected the temporally regulated aspects of expression of either the $\alpha^{\prime}$ or $\beta$-subunit promoters. The results of these studies have undergone several rounds of reviewers comments and will be published, with modifications, in Plant Molecular Biology during 1993. A copy of the manuscript is attached (Lessard et al).

In concurrent work $\mathrm{T}$. Fujiwara took a somewhat more defined approach and created site directed nucleotide changes in the RY element, and in the SEF 3 and SEF 4 protein binding sequences. After confirming that these changes altered the in vitro binding of the proteins to their respective sites, the modified promoters were introduced into transgenic plants. The results of that study are detailed in the manuscript by Fujiwara and Beachy (manuscript submitted for publication, attached) and demonstrated that there was no clear correlation between the in vitro binding assays and the expression of the genes in transgenic plants. Furthermore none of the mutations in the SEF 3 and 4 binding sites altered the levels of gene expression or the temporally regulated pattern of gene expression. The unexpected result of these studies was that mutations in the RY sequence alement resulted in loss of tissue specificity of gene expression; the gene was expressed in leaf tissues. This occurred when the mutated element was associated with the core of the CaMV 35 S promoter, but not with the core promoter of the $\alpha^{\prime}$ promoter.

The promoters of seed storage protein have significant potential applications in biotechnology. In a demonstration of one such application Fujiwara carried out a study to demonstrate seed specific repression of GUS activity in tobacco plant by antisense RNA. Here we demonstrated conclusively that antisense RNA complementary to the GUS mRNA was produced in transgenic plants. When the test plants also expressed a GUS gene in the $(+)$ sense under control of the CaMV 35S promoter, the amount of GUS enzyme produced in embryos was greatly reduced compared to those that did not carry the (-) sense gene construct. This work was reported by Fujiwara and Beachy (reprint enclosed)

Fujiwara also completed a study which confirmed the results of a previous study from this laboratory demonstrating that the enhancing activity of the $\alpha^{\prime}$ promoter sequences enhanced the expression of the $\beta$-subunit promoter when the promoters were in proximity with regard to each other.(Naito et al. Plant Molec. Biol 11,109-123; 1988). While there was clear enhancement of gene expression, there was not effect on the temporal pattern of gene expression of either the $\alpha^{\prime}$ or $\beta$-gene promoters. These results are reported in a paper by Fujiwara and Beachy (1993; attached). 
Page 3 of 5

\section{WORK IN PROGRESS}

In previous studies supported by this grant, we reported that expression of the $\beta$-gene but not the $\alpha^{\prime}$ gene was affected by ABA. Lessard, during his graduate studies at Washington University, studied the effects of adding $A B A$ to developing seeds of transgenic tobacco plants that expressed GUS genes driven by deletion mutants of the $\beta$-gene promoter (P. Lessard, Ph.D. Thesis, Washington University, 1991). The genes that were introduced and expressed in tobacco plants have also been used, in ongoing collaborations with S. Naito and T. Fujiwara (Hokkaido and Tokyo Universities, respectively) in transgenic Arabidopsis thaliana. These plants are currently being crossed with a mutant biovar of $A$. thaliana that does not produce $A B A$. The results of the studies to determine which $\beta$ gene promoters are affected by this mutant, resulting in loss of gene regulation will be determined during 1993.

During the past 14 months (since relocating the project to The Scripps Research Institute) our work (carried out experimentally by Dr. J. Sun, post-doctoral associate) has been directed in two additional areas that are briefly described here:

(1) cloning the gene encoding the SEF 3 and 4 proteins. Several large cDNA libraries were constructed in a protein expression phage-derived vector using as templates mRNAs isolated from developing soybean embryos. While these libraries have been very large, the screening to date has not yielded a positive result, i.e., we have not isolated a cDNA that encodes a known SEF 3 or SEF 4 protein. The major problem has been that screening with oligo nucleotides or DNA fragments that contain the SEF 3 and SEF 4 binding sites have yielded considerable background contamination. Thus, on subsequent rescreening with mutant oligos or DNA fragments, the putative positive clones were eliminated from the screening process. At the current time Dr. Sun is working with a nuclear extract prepared from embryos on a proteinDNA assay ('southwestern') to attempt to identify a specific protein on a blot assay. Perhaps such an approach will lead to a different strategy for cloning these DNA binding proteins. As described above and in the attached manuscripts, we remain convinced that these proteins are, under some conditions, involved in the expression of the $\beta$-conglycinins. However, the manner in which they do so remains elusive. We will continue to attempt to characterize these proteins, but have also diverted significant effort to achieving other stated goals of the project. 
2) As we described in earlier publications, and in the Fujiwara and Beachy paper (1993; attached) the $\alpha^{\prime}$ and $\beta$-genes are expressed with different temporal and spatial patterns. This has proven to be the case when either the cognate coding sequences or the GUS reporter gene are used. We have constructed a number of different chimeric genes to attempt to identify the promoter/or downstream sequences that are involved in distinguishing the expression patterns of these two gene promoters. These studies include: constructing chimeric promoters that place the core promoters of each gene with upstream sequences of the opposite gene, keeping intact the 3 non-translated region of the $\alpha$ gene. In one set of gene constructions the SEF 3 binding site, which is found in the $\alpha$ but not the $\beta$-gene, was introduced at the appropriate relative position into the $\beta$ gene promoter, and the chimeric promoter was ligated to the GUS sequence. To address the possibility that differences in gene expression reflect differences in mRNA stability as suggested several years ago by Harada et al ( $R$. Goldberg's group) and Naito et al (this lab) genes were constructed that exchanged the non-translated $3^{\prime}$ ends of the $\alpha^{\prime}$ and $\beta$-genes with the $\alpha^{\prime}$ and $\beta$-gene promoters, using the GUS coding gene sequence as a reporter. The constructs described here have/or will soon be introduced into a plant transformation vector and are being used to develop transgenic tobacco plants.

3) Continuing our interest in the biotechnological implications of the seed promoters we have established collaborations with several laboratories in the San Diego area to study some interesting biological reactions. In the first (currently in progress) we will express a potent ribosome inactivating protein (RIP), 'saporin', during embryo development. The gene construct contains, or lacks, a signal peptide that should determine whether the protein is targeted to the vacuoles/protein bodies, or is cytoplasmic. We expect that one or more of the constructs will result in embryo abortion and the other will not. A local biotechnology company is targeting the use of this RIP in an immunobased cell killing product: if our work to target the protein to protein bodies is successful (i.e., the embryos are not killed) we expect to collaborate with this company to express the immunotoxin in seeds.

The second collaboration involves members of the Molecular Biology, Cell Biology, and Chemistry Departments here at TSRI that have developed genes encoding either potential therapeutic antibodies, or catalytic antibodies. During the next year we will construct transgenic plants (tobacco, and rapeseed) that will express these gene under control of the $\alpha^{\prime}$ gene promoter. We will determine the levels of expression and protein functionality (for the catalytic $A b$ ) and degree of accumulation and stability (for all $A b$ ). 

Government. Neither the United States Government nor any agency thereof, nor any of their employees, makes any warranty, express or implied, or assumes any legal liability or responsibility for the accuracy, completeness, or usefulness of any information, apparatus, product, or process disclosed, or represents that its use would not infringe privately owned rights. Reference herein to any specific commercial product, process, or service by trade name, trademark, manufacturer, or otherwise does not necessarily constitute or imply its endorsement, recommendation, or favoring by the United States Government or any agency thereof. The views and opinions of authors expressed herein do not necessarily state or reflect those of the United States Government or any agency thereof.

Roger N. Beachy

SCRIPPS RESEARCH INSTITUTE

La Jolla, CA 92037

Genetic Engineering with a Gene Encoding a Soybean Storage Protein R.N. Beachy, Department of Cell Biology

The $\beta$-conglycinins are soybean storage proteins encoded by genes that are tightly regulated both spatially and temporally. We have studied the Soybean Embryo Factors that bind to the cis elements that are presumably involved in regulating the expression of these gene promoters using both in vitro binding assays and in vivo expression assays in transgenic plants. The results obtained to date have made it evident that there are no clear correlations between the in vivo and the in vitro results, i.e., changes in single nucleotides that alter protein:DNA interactions can have little or no impact on expression of the promoters in vivo. In contrast, the CATGCAT (RY element) sequence, for which no binding proteins have been identified, appear to be very important for controlling gene expression. Although we have been attempting to isolate and characterize the SEF 3 and SEF 4 proteins we have to date not been successful using protein expression libraries derived from embryo cDNAs. We are continuing experiments of this type, as well as more standard protein purification procedures.

We have constructed a number of chimeric promoters with different upstream and downstream regulatory sequences in an attempt to identify, by expression assays in transgenic plants, those sequences that are uniquely responsible for the temporal and spatially regulated expression of the $\beta$-conglycinin genes. Because of the results of previously published work from this laboratory, we concluded that the core promoters themselves may be responsible for the regulation. Therefore, we have focused our efforts on these sequences, the RY element, and the SEF 3 binding sequences. In a follow-up to our studies to modify the expression of genes in seeds, we will express several types of human and other animal genes in seeds of transgenic plants. 

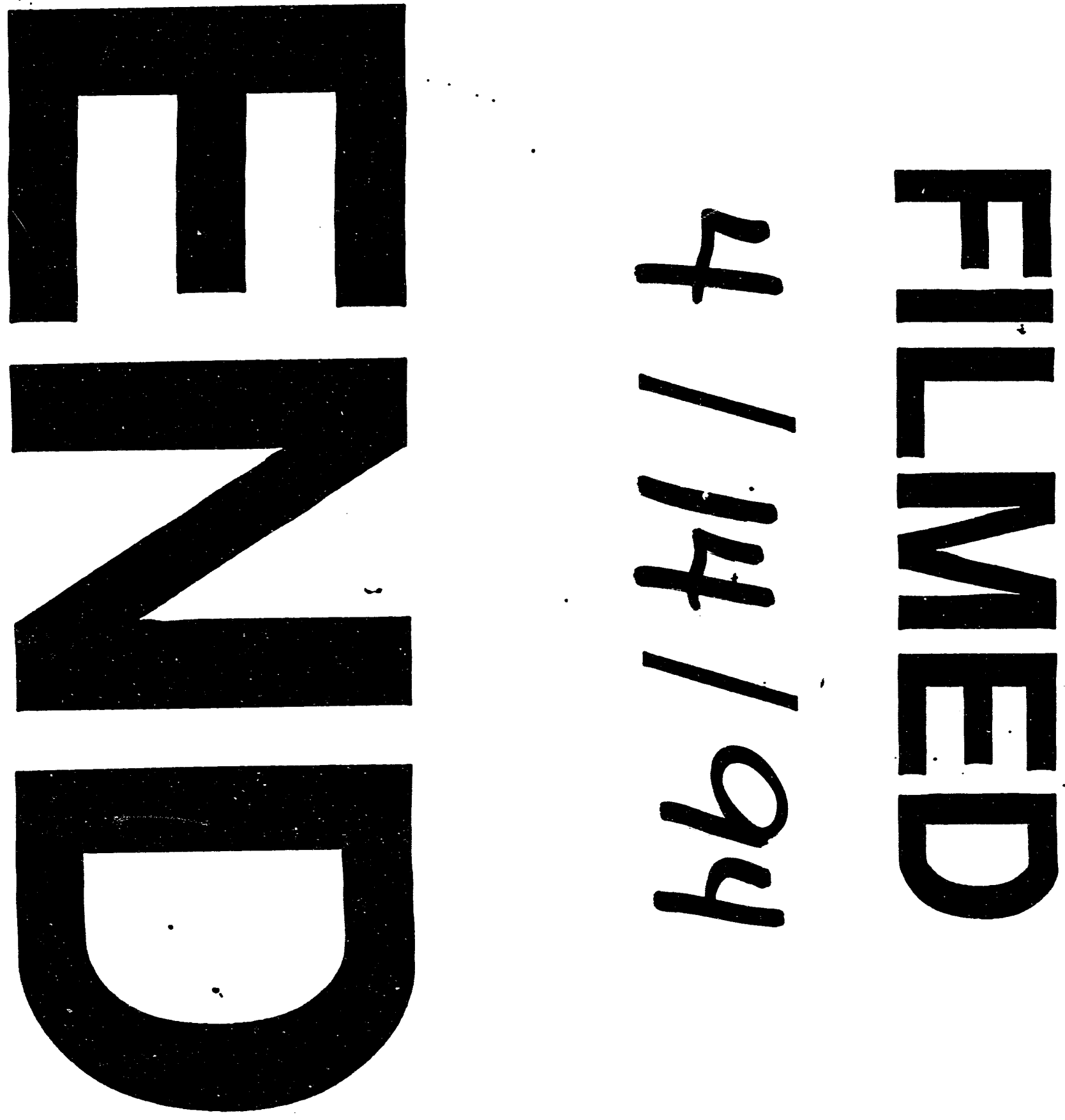
$\geq 0$

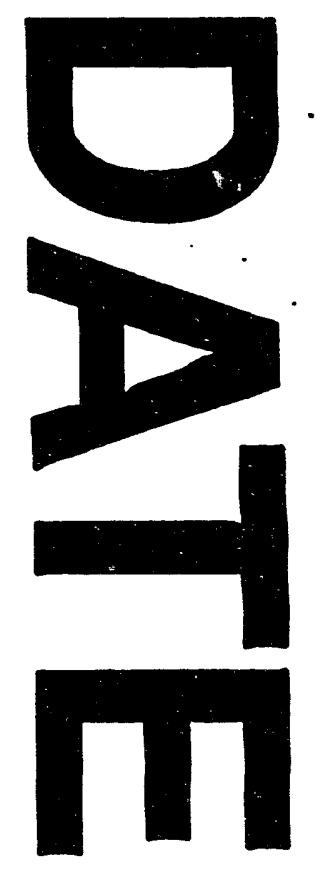


